\title{
Spoke ring and anchorage of nuclear pore complex revealed by high resolution transmission electron microscopy
}

\author{
Jarmil Prachař \\ Laboratory of Tumor Immunology, Cancer Research Institute, Slovak Academy of Sciences, Vlárska 7, 83391 Bratislava, \\ Slovak Republic
}

\begin{abstract}
Using several different methods, the nuclear pore complex (NPC) was shown to be anchored in the nuclear envelope into the specific curved region, called pore membrane. Three transmembrane nucleoporins in the equatorial region of NPC contain hydrophobic stretches, which exhibit the ability to intersect the phospholipid bilayer. Using transmission electron microscopy, we observed three different evaluable morphological situations in the section through the NPC spoke ring (SR). We suppose that some sections are directed through one type of subunit that is responsible for anchoring. Other sections are directed through the second type of subunit that may provide pore membrane bending. Finally, the spoke ring is sectioned between aforementioned subunits where the pore membrane is best preserved. The proposed anchor is represented by the chains of protein complexes which replace phospholipid bilayer in a relatively large area. Second subunit, presumed bending module is represented by the bundles of chains copying the shape of the pore membrane from the side of the NPC. This work is based on very high resolution resulting in unique and complicated images of tangled and cut off protein chains, nevertheless, it provides insight into how some proteins interact with or replace the membrane.
\end{abstract}

Key words: Nuclear pore complex — Spoke ring - Anchorage - High resolution transmission electron microscopy

Abbreviations: NPC, nuclear pore complex; SR, spoke ring.

\section{Introduction}

There are at least two ways how the material is exported from the nucleus to the cytoplasm. One way, recently found, is budding of nuclear membranes, which pack large ribonucleoproteins to be exported to the cytoplasm (Speese et al. 2012). The second and major way, known for decades, is export of many different cargos through the nuclear pore complexes (NPCs). Besides export, it is the import of all material needed for nuclear functions that is believed to be provided by the same NPCs. NPCs are large proteinous complexes enabling swift exchange of very different material ranging from small molecules to large ribonucleoprotein

Correspondence to: Jarmil Prachař, Laboratory of Tumor Immunology, Cancer Research Institute, Slovak Academy of Sciences, Vlárska 7, 83391 Bratislava, Slovak Republic

E-mail: jarmil.prachar@savba.sk complexes in a selective manner (Wente 2000; Weis 2002). Both the inner and outer nuclear membrane bend and join together to create pores. The curved part of the membrane surrounding the pore opening is called pore membrane. NPCs are embedded in pores.

It is generally accepted that the major NPC anchor is in the equatorial plane in the pore membrane. Nucleoporins enabling this anchorage were identified and characterized in metazoan and yeast (Alber et al. 2007a, 2007b; FernandezMartinez and Rout 2012; Löschberger et al. 2012).

The assigned positions of these proteins reveal that they communicate by one part with the proteins of the spoke ring (SR), while the other part directs into the lumen of the nuclear envelope. Hydrophobic stretches, in the model drawings, are shown as lines perpendicularly intersecting the phospholipid bilayer of the pore membrane (e.g. Amlacher et al. 2011).

Here, we present very high resolution micrographs where individual protein chains, comprising the several 
nanometer thick surface layer of the NPC section, are discerned.

Our results indicate that NPC anchorage is implemented by replacement of certain areas of the pore membrane by a complex of protein chains protruding from SR. At other sites, the SR does not attack the pore membrane. Moreover, at another sites, protein chains copy the shape of the pore membrane including its parts above and below the SR. This part of the SR might serve for bending the pore membrane like a clip or these regions might represent connections to the cytoplasmic and nuclear rings (Bui et al. 2013) and serve as a strong pillar for membrane bending.

Our observations reveal that the SR is not a simple ring, in terms of its height and generally the entire shape. The SR or its associated structures could accommodate even large complexes in specific vertical (or skew) positions. In the region of the NPC SR, the protein chains are intertwined into complicated tangles. These observations are new and striking. From this point of view, it is interesting to compare these high resolution micrographs with simply looking colored models of the inner ring published recently (Amlacher et al. 2011, Fig. 7). High resolution electron micrographs showing mostly cut-away protein chains are not in contradiction to or in agreement with the published models (Alber et al. 2007a, 2007b; Amlacher et al. 2011; Bui et al. 2013), they just show the NPC from different perspective not easily comparable at the present stage to published data.

In addition to the pore membrane, we have recorded how the nuclear and mitochondrial membranes are also interwoven with protein chains or even replaced by protein complexes.

\section{Materials and Methods}

\section{Cell cultures}

Mouse leukemia cell line L1210 (purchased from the American Type Culture Collection, Rockville, MD, USA) was maintained in RPMI 1640 medium supplemented with $10 \%$ fetal bovine serum. The cells were fixed at a cell density of $10^{6} / \mathrm{ml}$ while still dividing, yet after the exponential growth phase.

The human colon adenocarcinoma cell line Caco-2 was obtained from the European Collection of Cell Culture (Wiltshire, UK). The cells were cultured in RPMI 1640 medium supplemented with $10 \%$ fetal bovine serum and collected for electron microscopy study in the early exponential growth phase. The cells adhere very strongly to plastic, therefore glass dishes were used for cultivation to prevent loss of the bottom cellular membrane. The cells were scraped following osmium tetroxide postfixing. The Caco-2 cells were described in detail earlier (Prachar 2010).
Preparation of samples for transmission electron microscopy

The L1210 cells ( $5 \mathrm{ml}$ ) were quickly pelletized $(2 \mathrm{~min})$ and immediately fixed without any washing. The Caco- 2 cells were treated in the same way as the leukemia cells, yet after the postfixing step (see above). Fixation, contrasting, embedding and staining of sections were done by conventional procedures. Briefly, the procedure was as follows: fixation with 3\% glutaraldehyde in PBS for $1 \mathrm{~h}$, postfixation in $1 \%$ $\mathrm{OsO}_{4}$ for $1 \mathrm{~h}$, washing 3 times with cold deionized water, and contrasting with a saturated solution of uranyl acetate in deionized water overnight. For washing, cells were resuspended in water and shortly centrifuged 3 times between each step. Subsequently, the cells were resuspended in low melting point agarose $(1 \%$ in water $)$ at $37^{\circ} \mathrm{C}$, pelletized and kept in an ice bath for a short time, required for embedment in agarose. The following step was dehydration in $70 \%$ and $100 \%$ ethyl alcohol and equilibration in propylene oxide. Embedding was done in Poly/Bed 812 epoxy resin (Polysciences). For preparation of the epoxy resin, a combination of components was chosen to achieve maximal hardness, according to the manufacturer's instructions. The sample in epoxy resin was kept at $37^{\circ} \mathrm{C}$ for $48 \mathrm{~h}$ to harden, and at $50^{\circ} \mathrm{C}$ for the next $24 \mathrm{~h}$. Ultrathin sections were picked up directly on bare 700 or 1000 mesh copper grids (Agar Scientific). Section thicknesses were about $50 \mathrm{~nm}$ or less. Contrasting of the ultrathin sections (one side only) was done on a drop of a saturated solution of uranyl acetate in water at $37^{\circ} \mathrm{C}(2 \mathrm{~h})$ and, after intensive washing in deionized water, the last step was contrasting on a drop of Reynolds solution at laboratory temperature (4 min or less). Unless otherwise stated, all procedures were carried out at room temperature. Uranyl acetate and Reynolds solution were prepared the same day as contrasting was done.

Images were collected using the JEOL JEM-1200 EX transmission electron microscope at $80 \mathrm{kV}$ with Kodak electron microscope films 4489.

\section{Handling the microscope to attain high resolution in cell sections}

In search for the object of interest and for focusing, the usual microscope setting was used. The spot size number used was 2 or 3 (measured diameters of these spot sizes in our microscope are $2 \mu \mathrm{m}$ and $1.5 \mu \mathrm{m}$, respectively). This setting provides sufficient illumination of the screen to search for and focus on the objects in binocular magnifier with zoom $\times 10$ and there is a minimal risk of radiation damage. After focusing, the beam current was switched off, the brightness was set to underfocus position (counterclockwise) and the setting of spot size was changed to number 5 (diameter $500 \mathrm{~nm}$ in our JEOL JEM 1200 EX microscope). We waited $15 \mathrm{~min}$ in this step for stopping any specimen movement 
(drift) in case of very thin sections. Further, the beam current was retrieved to the saturated position. Thereafter, from the dark position, the brightness was slowly and carefully set to long exposure (usually up to $90 \mathrm{~s}$ for film KODAK 4486 with set sensitivity SE 6 - it causes certain overexposure; current density was in our setting $1.5 \mathrm{pA} / \mathrm{cm}^{2}$ or more). The alignment of the microscope for spot size 2 and 5 must be carefully performed to obtain the same center. The underfocus mode was used for the second condenser lens, which regularly means that to reduce brightness the brightness knob is turned counterclockwise from the crossing. But here, we started in reverse direction from the dark uderfocus position to the first registered current density (clockwise turning of the brightness knob). Therefore, the second condenser lens worked in a weak mode, electron trajectories are close to parallel and aberrations of this lens are minimal.

\section{Results}

We present here several sections through NPCs close to the NPC axis perpendicular to the envelope plane and we analyze three representative ones in detail. Consequently, we characterize in detail six sections through the pore membrane and through the SR.
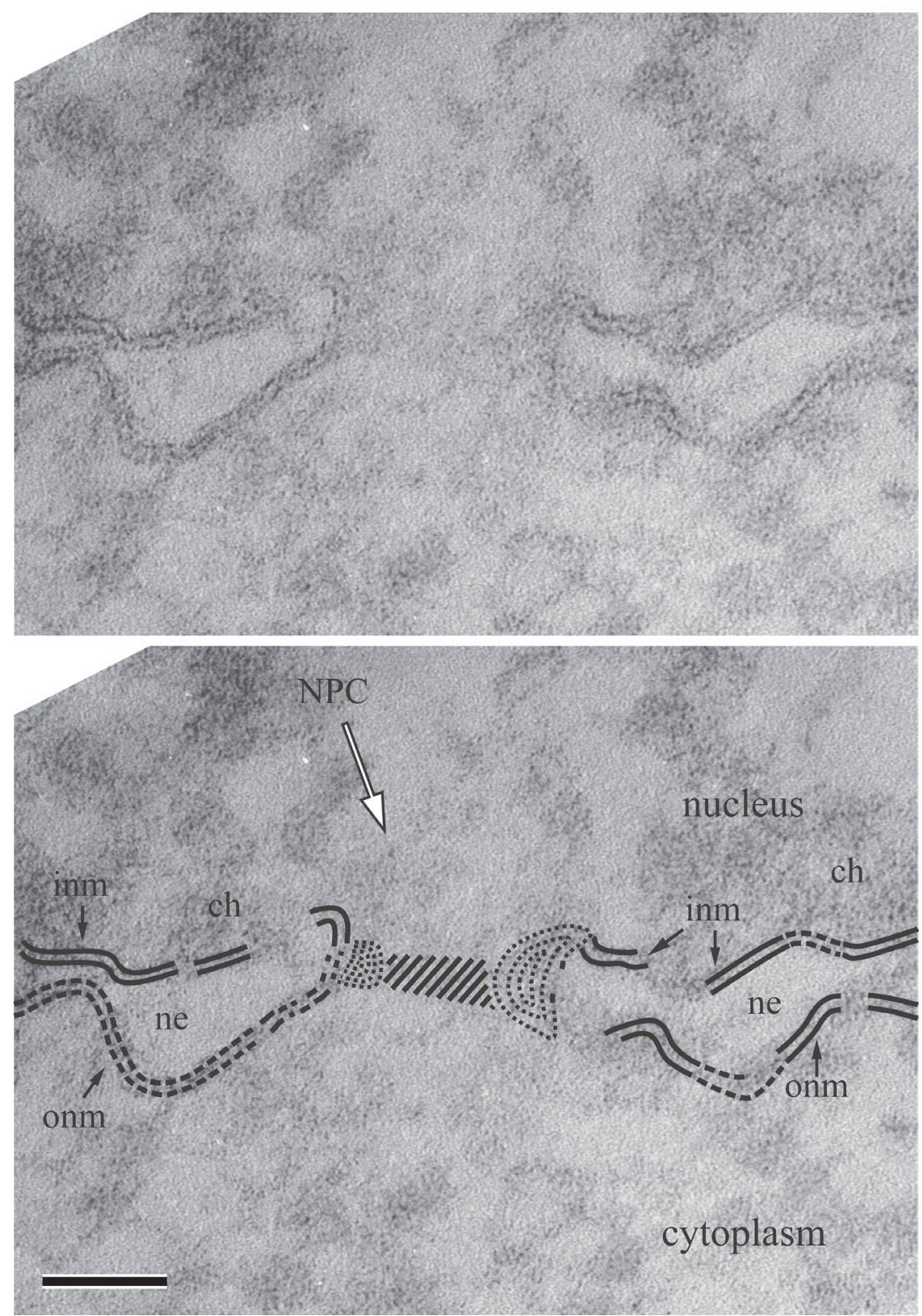

Figure 1. Very thin section through the NPC of L1210 cell. Features of interest are depicted in the lower part of the figure. White arrow directs to the center of the NPC. Outer nuclear membrane (onm), inner nuclear membrane (inm) and pore membrane are outlined by continuous or dashed lines. The spoke ring is depicted by dotted lines and the barrier is crosshatched. Note the insertion of heterochromatin into the inner membrane, close to the right edge of the NPC. ch, heterochromatin; ne, nuclear envelope. Original magnification: $\times 150,000$; scale bar: $50 \mathrm{~nm}$ (reprinted from Prachař 2012, Fig. 5, with permission from Elsevier). 

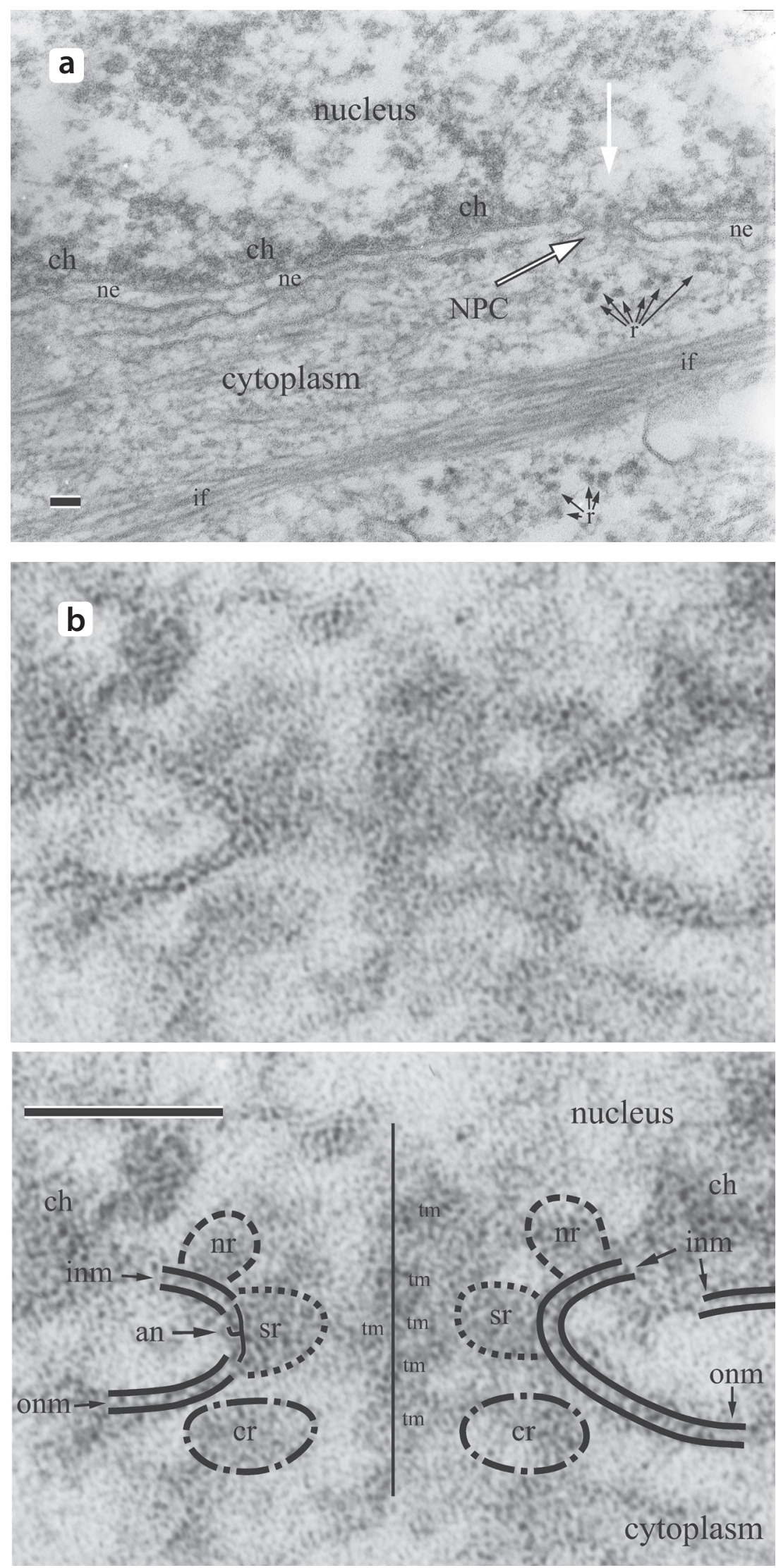

Figure 2. One representative NPC in section through metabolically highly active Caco2 cell. a. Larger area of cytoplasm, nucleus and nuclear envelope with one NPC. White arrow, without outline, in the axis of NPC directs to the electrondense particles, which presumably represent transported material (reprinted from Prachař 2012, Fig. 8, with permission from Elsevier). b. An enlargement of Fig. 2a showing primarily the NPC. Features of interest are depicted in the lower part of the figure. The NPC axis is depicted as a thin solid line. The outer nuclear membrane (onm), inner nuclear membrane (inm) and pore membrane are outlined by continuous lines. Nuclear and cytoplasmic rings are anticipated only on the basis of their positions and on comparison with published cryotomography data (Maimon et al. 2012). Some protein chains serving for anchorage are outlined with thin solid line. Note the insertion of heterochromatin into the inner nuclear membrane, close to the right edge of the NPC (similar to that shown in Fig. 1). an, anchorage of NPC into the pore membrane; $\mathrm{ch}$, heterochromatin; $\mathrm{cr}$, presumed cytoplasmic ring; if, intermediate filaments; ne, nuclear envelope; nr, presumed nuclear ring; r, ribosomes; sr, expected spoke ring; tm, transported material near NPC axis. Original magnification: $\times 60,000$; scale bars: $50 \mathrm{~nm}$. 
A very thin section through the NPC is presented in Fig. 1. The SR is not well distinguished in this micrograph because the contrast is too low. Nevertheless, on the left edge of the NPC we can discern a relatively well preserved nuclear membrane, which is not affected by the SR. On the right edge of the NPC, the SR seems to be robust in comparison with the left edge and the pore membrane is not well preserved. This might represent specific situation where the section goes through presumed bending module on right side and between two subunit types on the left side. This micrograph also serves for comparison with the following observations.

An overview around one NPC in a highly proteosynthetically active Caco-2 cell is shown in Fig. 2a. Material transported along the central axis of the NPC is well discernible. Enlargement of the NPC presented in Fig. $2 \mathrm{a}$ is shown in Fig. 2b. On the right side, the pore membrane is well preserved, while the pore membrane on the left NPC edge is interwoven by presumed protein molecules coming out from the SR. This site might serve for anchorage of the NPC. Interestingly, close to the right edge of the NPC, the inner nuclear membrane is not continuous; the membrane is interrupted by heterochromatin, similarly as in the case of the NPC shown in Fig. 1.

The anchorage of the NPC in the pore membrane is documented at higher resolution in Fig. 3a and enlarged in Fig. 3b. A complex of protein molecules replaces part of the pore membrane on the left side of the NPC section. At high enlargement shown in Fig. 3b, the membranes are not simple bilayers but are interwoven very often with protein molecules. These molecules are not distinguishable in lower resolution or magnification. The protein chains are white or very light, while dark heavy metals are situated around them. The reason for this unusual view is high resolution combined with very low depth of field. We observe practically only the surface of the section; the section surface was also used for focusing. The membranes presented as discontinuous lines of phosphate heads are found much more often than two continuous dark lines, but places with "classical" two dark lines, well known from textbooks, can also be found (Fig. 3d). A very similar situation of potential anchorage documented in Fig. 3a and 3b was shown in Fig. 2b, yet better distinguished in Fig. 3b, due to higher magnification and resolution. The height of the anchorage complex appears to be about $15 \mathrm{~nm}$ only. Examples of much larger complexes replacing the membranes in a much larger area are documented in Fig. 3e and 3f.

On the right side of the NPC section shown in Fig. $3 \mathrm{a}$ and enlarged in Fig. 3c, is a view similar to that on the right side of the NPC section in Fig. 1. The protein chains are tangled around the pore membrane and they continue up and down from the region of the SR. These complexes copy the shape of the pore membrane. The pore membrane is more or less maintained on the nuclear side. The cytoplasmic side of the pore membrane is composed of crisscrossed chains and it is not possible to determine the position of membrane lipids. The SR region shows a denser chain network than the regions on the cytoplasmic and nuclear side. Interestingly, the whole right edge of the NPC shows relatively strong staining with heavy metals, indicating the presence of negative charges, for example phosphate groups or acidic amino acids. The protein chains seem to be branched but the cause of such a view can be the sectioning of packed protein complexes.

\section{Discussion}

\section{General analysis of observations}

One section through the center of the NPC provides two sections through the pore edge. We analyzed six sections through the pore membrane and SR in detail in this work. This number of observations is insufficient for quantitative analysis. Nevertheless, the situations documented in sections through three independent NPCs reappeared. This may be explained by the assumption that there are not many different morphological situations in this small region. Indeed, the three different morphological states would be sufficient for explaining the supposed functions of the NPC SR as follows. One type of subunits of the NPC scaffold arranges anchorage; the other type of subunits provides the bending of the pore membrane. The pore membrane would be best preserved with minimal interference between these two regions. This speculation is based on the assumption that there are at least two types of functional subunits in the rings. Indeed, two types of subunits in the number eight and eight have been predicted for every NPC subring (Akey and Radermacher 1993; Alber et al. 2007b; Amlacher et al. 2011; Aitchison and Rout 2012). Such subunits need not to be distinguished as individual morphological entities even on using high resolution; they might spatially merge with neighboring subunits. On the other hand, an underlying 16-fold symmetry of the NPC has been suggested in earlier works (Unwin and Milligan 1982; Akey and Radermacher 1993; Yang et al. 1998). On the level of the cytoplasmic ring, the presence of twice more than eight morphologically distinguishable subunits was also reported in 1996 (Goldberg and Allen 1996). In the same work, one can see that triangular units of the nuclear ring alternate with bases of nuclear basket struts. This situation can therefore be interpreted also as eight subunits alternating with different eight subunits. On other levels of the NPC, the sixteen morphologically distinguishable subunits cannot be found (Maimon et al. 2012). Nevertheless, different methods showed unequivocally sixteen subunits on all levels of the NPC (Alber et al. 

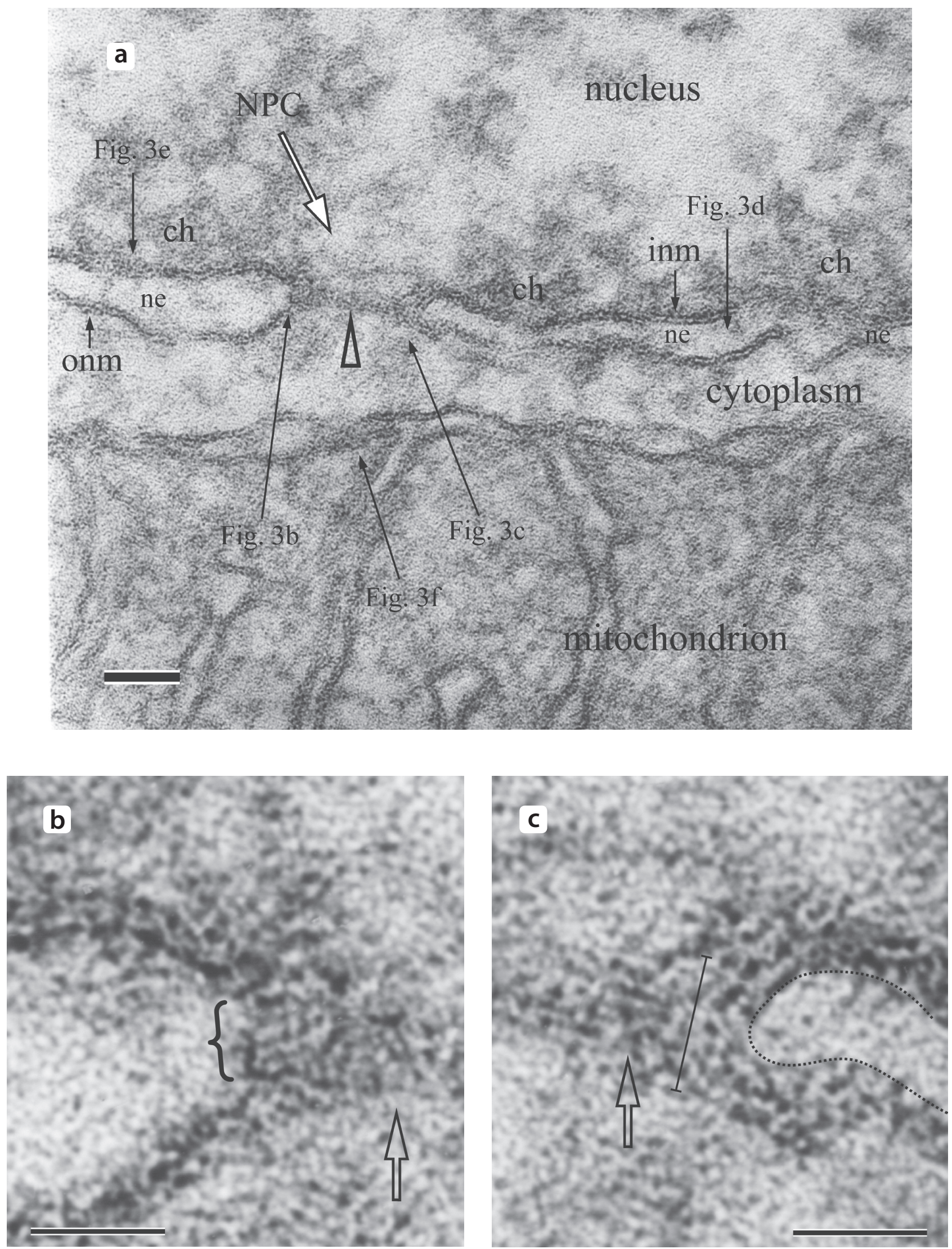

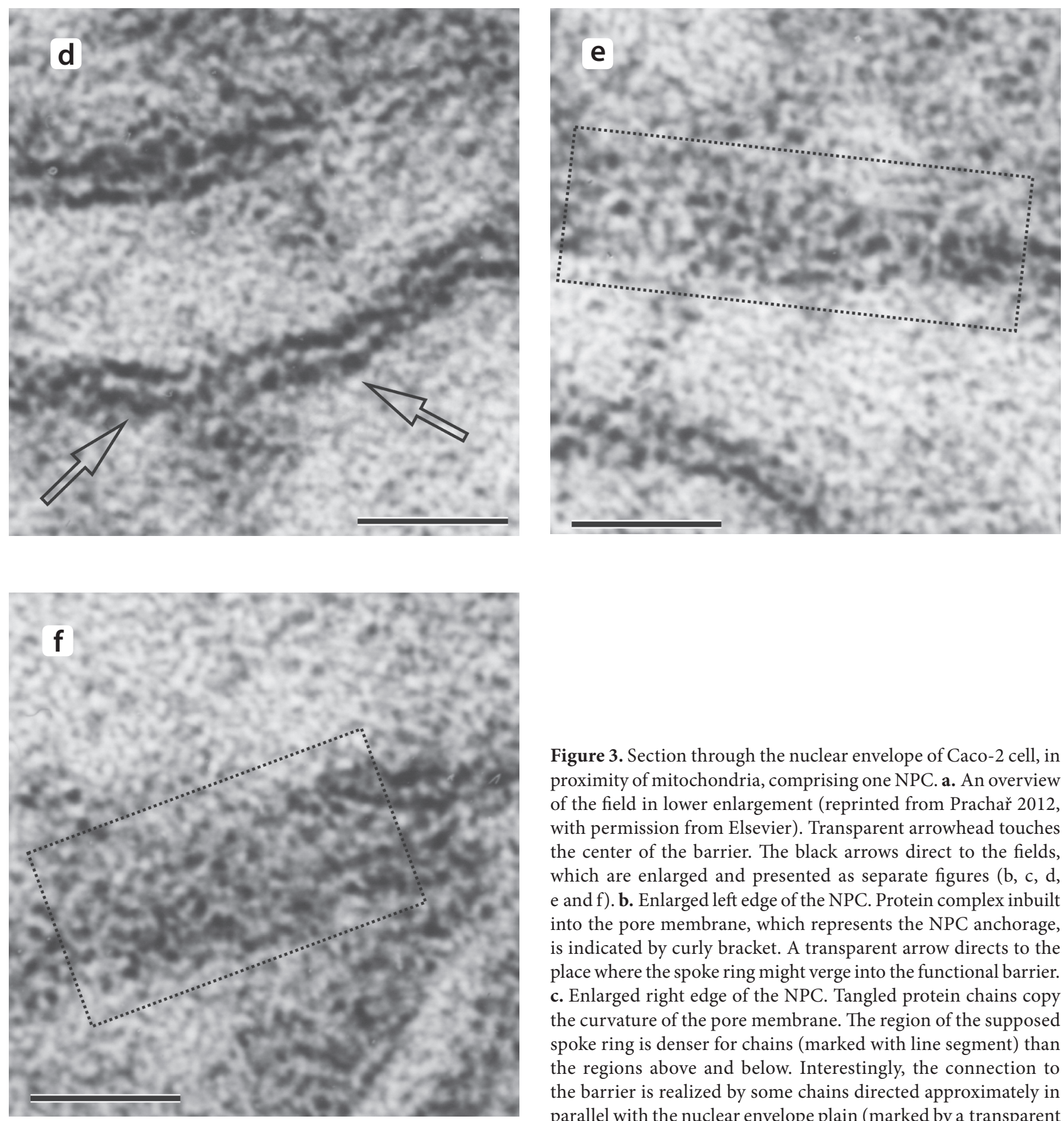

Figure 3. Section through the nuclear envelope of Caco- 2 cell, in proximity of mitochondria, comprising one NPC. a. An overview of the field in lower enlargement (reprinted from Prachař 2012, with permission from Elsevier). Transparent arrowhead touches the center of the barrier. The black arrows direct to the fields, which are enlarged and presented as separate figures (b, c, d, e and f). b. Enlarged left edge of the NPC. Protein complex inbuilt into the pore membrane, which represents the NPC anchorage, is indicated by curly bracket. A transparent arrow directs to the place where the spoke ring might verge into the functional barrier. c. Enlarged right edge of the NPC. Tangled protein chains copy the curvature of the pore membrane. The region of the supposed spoke ring is denser for chains (marked with line segment) than the regions above and below. Interestingly, the connection to the barrier is realized by some chains directed approximately in parallel with the nuclear envelope plain (marked by a transparent arrow). The perinuclear space is marked by a dotted line. d. The phospholipid membrane that show the classical composition as a white line between two dark lines. These segments are not interwoven with protein chains (except presumable ribozome anchorage), yet they are relatively rare in the case of nuclear membranes in high resolution views. Two transparent arrows direct to this "classical" view of the membrane. Interestingly, between the inner and outer nuclear membrane tangles of molecules, presumably proteins, can be distinguished (see also Fig. 3b, $\mathrm{c}$ and e). The presence of this matter is well justified for retaining both membranes at appropriate distance. e. The insertion of large proteinous complex (or heterochromatin) into the inner nuclear membrane (marked by dotted rectangle). f. Large proteinous complex replaces both, the inner and outer mitochondrial membrane (marked by dotted rectangle). Note that this complex is situated opposite the center of the NPC potentially allowing a connection between the nucleus and the mitochondrial matrix. ch, heterochromatin; inm, inner nuclear membrane; ne, nuclear envelope; onm, outer nuclear membrane. Original magnification: $\times 100,000$; scale bar in Fig. 3a: $50 \mathrm{~nm}$, scale bars in Fig. $3 \mathrm{~b}$ to $3 \mathrm{f}: 20 \mathrm{~nm}$. 


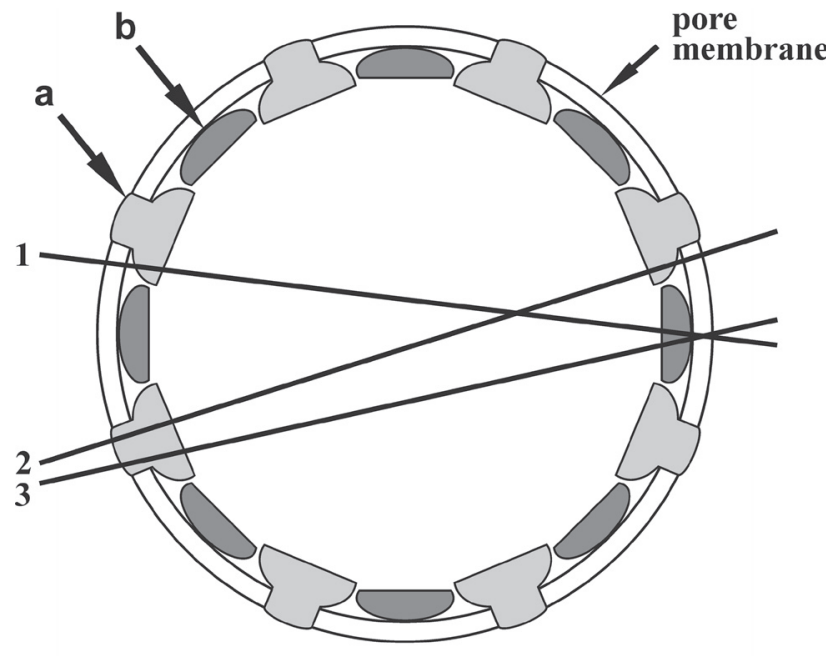

Figure 4. Hypothetical scheme of possible positions of sections through the NPC. The drawing shows a bird's eye view on the NPC SR, thus the equatorial NPC plane. The hypothetical subunits of SR provides anchoring (a) and pore membrane bending (b). The section 1 passes out of the NPC anchorage on the left side and through subunit $\mathrm{b}$ on the right side, representing the section presented in Fig. 1. The section 2 passes through the anchorage on the left side and out of the anchorage on the right side, representing the view presented in Fig. 2 b. The section 3 passes through the anchorage on the left side and through subunit $b$ through the bending module (see Fig. 3a, b, c). 2007a, 2007b). The presence of sixteen subunits explains ideally our observations.

An eightfold rotational symmetry of the NPC means that this structure must be composed at least from eight subunits in every ring or the multiple of eight subunits, with the requirement that each type of these eight subunit sets must be different. If the subunit sets are not different, symmetry would appear as the multiple of eight. The presence of sixteen subunits (eight alternating with different eight) is in a full agreement with all published data, including findings presented here. Based on the micrographs given in this work, Fig. 4 presents our idea concerning the positions of the sections through the NPC. The shapes and distribution of the spoke ring subunits were based on the work by Alber et al. (2007a). However, the illustration there is a bird's eye view of the whole NPC, not a view at the level of SR. Moreover, the model of Alber et al. (2007a) is based on yeast NPC data. It follows from different works based on the models of metazoan NPCs, using contemporary methods, that SR subunits are not morphologically distinguishable as 16 subunits but as 8 subunits (Goldberg and Allen, 1996; Maimon et al. 2012). The spatial merging and interlocking of two sets of subunits $(8+8)$ would explain these findings. We drew this hypothetical model with distinguished subunits to simplify the understanding of functionality.

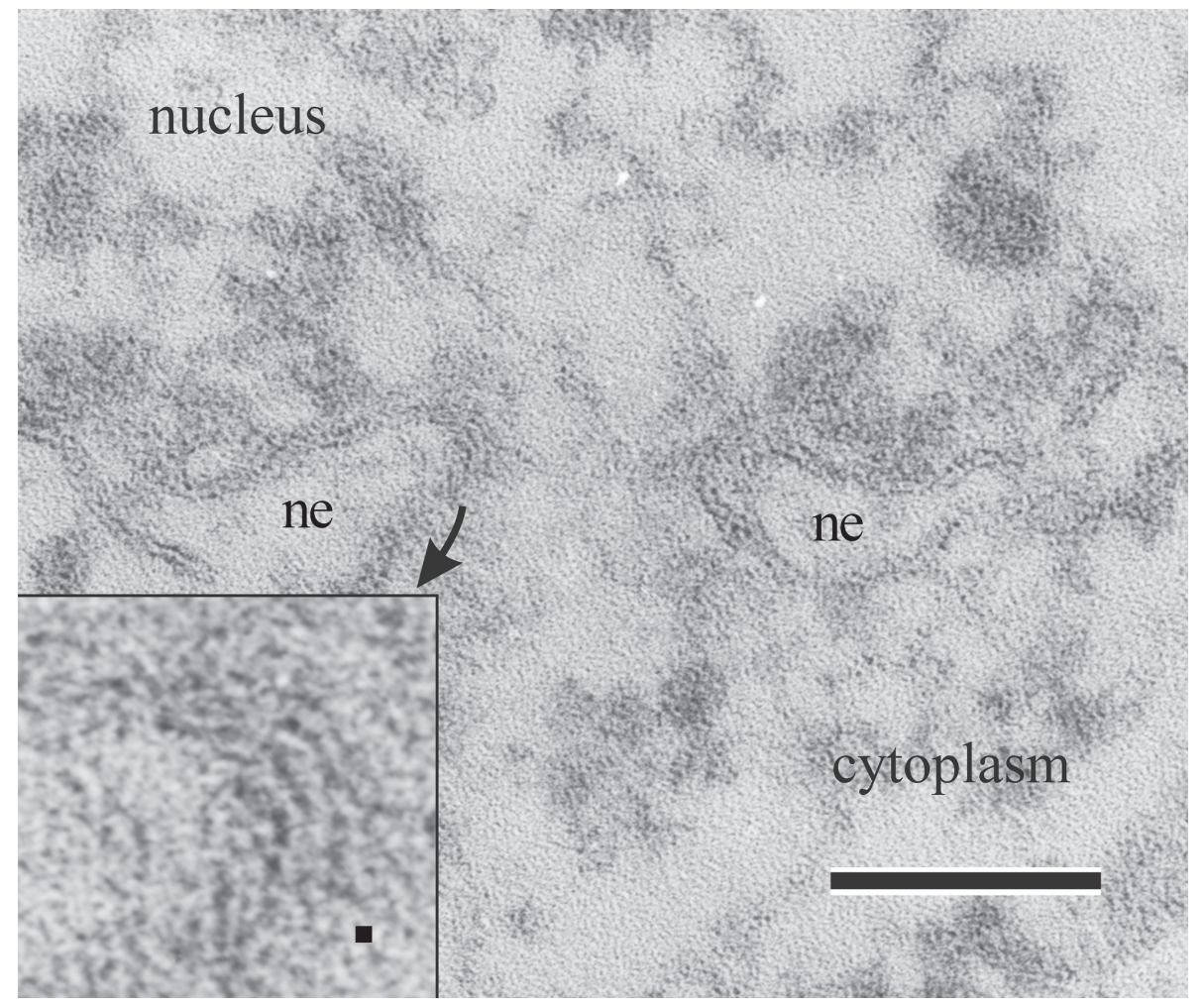

Figure 5. Representative micrograph of NPC section showing the pore membrane region like a structure of several layers. There is not possible to distinguish between phospholipid membrane and protein chains in such sections. This frequent observation can be the consequence of skew section and/or section directed not exactly through the NPC center. The inset represents the enlarged pore membrane region of the left-hand NPC edge. The object of observation is very thin section of L1210 cell. ne, nuclear envelope. Original magnification: $\times 75000$; scale bar: $100 \mathrm{~nm}$; small rectangle in the inset: $2 \mathrm{~nm}$. 


\section{Probability of presented observations}

From hundreds of inspected NPCs, only tens are sectioned perpendicularly to the nuclear envelope and approximately through the central NPC axis. Of these tens of NPCs only several show an evaluable section through at least one side. Thus the probability of findings presented here is very low. This low probability is presumably caused by technical reasons. The sections are probably not perfectly perpendicular to the envelope plane or are situated not exactly in the axis. High resolution emphasizes the effects of section inaccuracies. We chose three sections, where both the left and right side show evaluable situations. Sections shown in Fig. 5 occur more frequently. The pore membrane seems to be composed of several layers. Although such findings are not evaluable, they at least show that structures copying the shape of pore membrane do exist.

The most frequent findings show NPC as "classical" view, but they are not evaluable using high resolution, because the phospholipid membranes are not satisfactorily defined (Fig. 6). Inset in Fig. 6 is an enlargement of the pore membrane region in the right hand NPC edge. After further enlargement, it can serve for illustration of resolution higher than $1 \mathrm{~nm}$; various thin projections protrude from cut off residues of molecules.

\section{Micrographs resolution}

Three micrographs shown in Fig. 1, Fig. 2a and Fig. 3a presented in the section Results were already published in lower resolution, which was sufficient for characterizing the NPC barrier (Prachař 2012). We found that when positives are scanned using a high quality scanner at the resolution of 2400 dpi or more, the resultant picture can reach a resolution higher than one nanometer and subsequently protein chains become discernable. In the present study we show only some micrographs with the best resolution. For the quantitative analysis, more sections should be evaluated. In our earlier work, we published micrographs showing anchorage of mitochondrial nucleoid where chains of molecules intersected both the inner and outer mitochondrial membranes were presented (Prachar 2010, Fig. 3). At that time, we did not know that such high resolution of sections has never been published before. We continue to improve the resolution even further.

\section{Microscope setting}

The microscope setting used in this work means that we select the electrons close to the optical axis on both condenser lenses. The electron flow through the specimen is

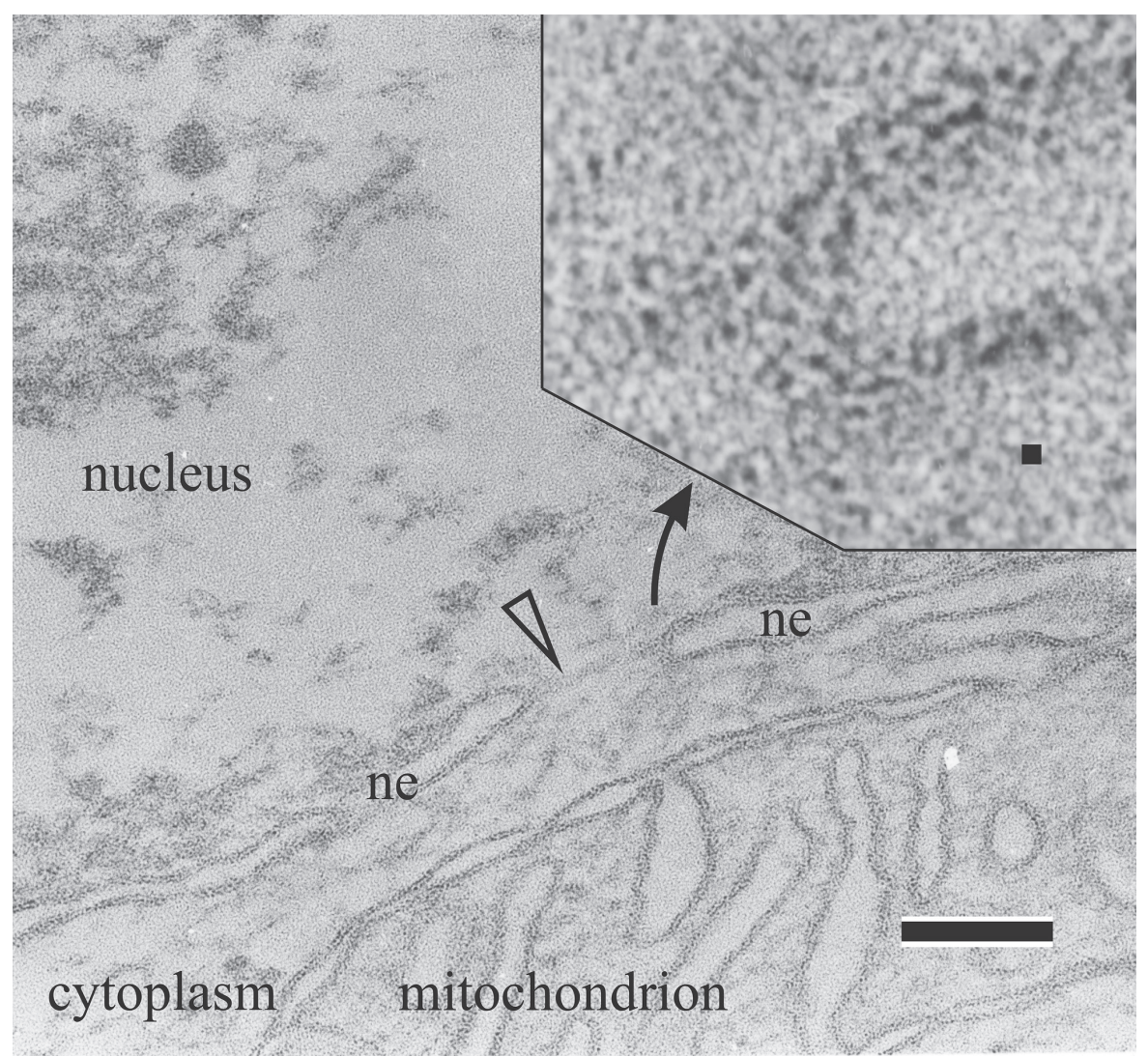

Figure 6. Very thin section through the nuclear envelope of L1210 cell, in proximity of mitochondria, comprising one NPC. This micrograph represents the most frequent observation of NPC in the cell section probably being skew. This "classical" view is not evaluable after enlargement in case of high resolution usage. The phospholipid membrane is not clearly distinguished as three lines (black, white, black) and it is not obvious whether protein chains of the spoke ring are inbuilt into the pore membrane or not (see Fig. 6 inset). The inset is the enlarged pore membrane region of the right-hand NPC edge. The thin barrier, marked by transparent arrowhead, was characterized in more details recently in Prachař (2012). ne, nuclear envelope. Original magnification: $\times 75000$; scale bar: $100 \mathrm{~nm}$; small rectangle in the inset: $2 \mathrm{~nm}$. 
weak and spread over a long time. Heat and charge have more time to disperse into the surroundings. For example, the selected diameter of the beam on the cathode going through the specimen in case of $90 \mathrm{~s}$ automatic exposure is 5 times smaller (closer to paraxial situation) than it would be in case of $3.6 \mathrm{~s}$ exposure. It is not important that we see only very weak illumination on the screen and we do not see the contours of the objects since the film is more sensitive than the human eye and consequently the film "sees" the object, which is already focused. Each electron incident on film is registered. If we tried to see the image well on the screen with spot size 5 set on the first condenser lens, the specimen would be immediately damaged by radiation.

In my opinion, it is very important that the electrons close to the optical axis (paraxial electrons) possess a very similar direction and especially very similar energy (this fact is not emphasized in any course of electron microscopy or in electron microscope manuals). The aberrations of lenses located below the specimen are much less manifested even if small apertures are not used. The drift of specimen is reduced or eliminated by using small meshed grids. Moreover, hardest epoxy resin was used for embedding. The result of this setting is also a very low depth of field and subsequently high resolution.

The selection of electrons with very similar energy and direction is a basic strategy of "physical" electron microscopes with atomic resolution.

The in-focus setting of the objective lens, which causes minimal phase contrast but maximal resolution, was used. Specimen surface was used for focusing.

Briefly, the strategy for obtaining high resolution without radiation damage is based on two different settings of the microscope. The first setting is used for searching and focus- ing the object, the second setting is used for photographing the object. The latter setting has unexpected powerful effect as to resolution.

It is probable that even higher resolution could be achieved by using a smaller condenser and objective apertures, and especially by using $\mathrm{LaB}_{6}$ cathodes which are stronger emitters than $\mathrm{W}$ cathodes, have smaller source spot size and thus electrons even closer to the optical axis could be selected. These approaches are, however, beyond our grasp. Usage of higher accelerating voltage than $80 \mathrm{kV}$ remains to be tested.

\section{Bending the pore membrane}

Several mechanisms of membrane bending have been published recently and they are subject of lively debates (see e.g. Kirchhausen 2012). The pores in the nuclear envelope originated by fusion of the inner and outer nuclear membrane. These membranes had to be bent to reach each other and then to fuse. Moreover, the shape of the pore membrane has to be maintained for NPC functionality. Structural analysis of several NPC proteins uncovered strong similarities between modules of coated vesicles and the NPC membrane-coating module as well as the structural evolution of this module (Devos et al. 2004; Debler et al. 2008; DeGrasse et al. 2009; Liu et al. 2012).

In vertebrates, the basic component and concurrently the largest building block of the NPC scaffold, is the Nup107160 complex. Recent data do not support the earlier "lattice" model where the supposed direction of Nup107-160 was parallel to the transport direction (Bilokapic and Schwartz 2012). Head to tail arrangement of Nup107-160, where these

\section{nucleus}

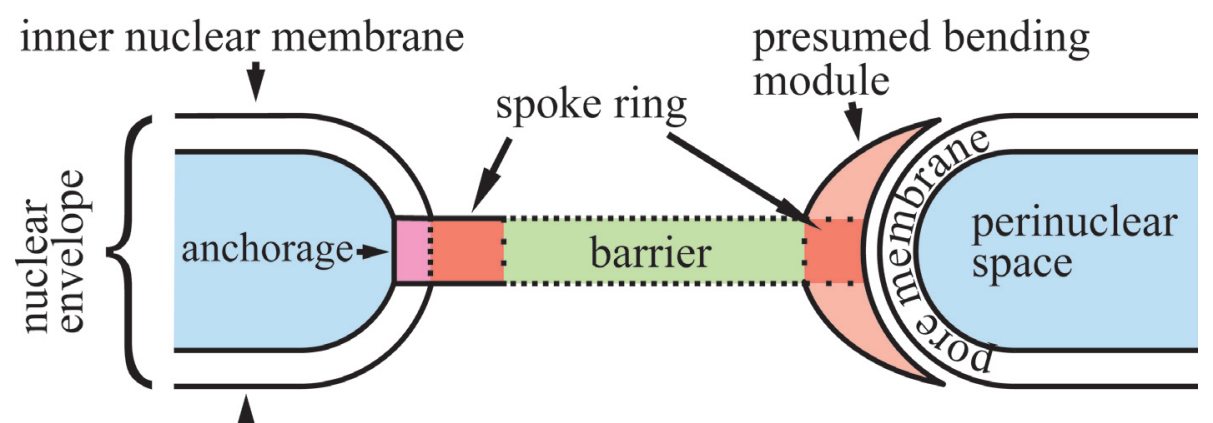

outer nuclear membrane

\section{cytoplasm}

Figure 7. Interrelation between nuclear pore complex and nuclear envelope. The scheme shows hypothetical simplified model of the NPC central part composed of spoke ring, barrier, presumed bending module and pore membrane. The anchorage of the spoke ring into the pore membrane is shown on the left pore edge (one of the eight proposed subunits). The spoke ring is not anchored by alternating eight subunits that juts up and down from the equatorial plane and these projections might serve for bending the pore membrane as schematically shown in the right pore edge. The barrier is anchored in the spoke ring and is of similar small thickness like the spoke ring (see Fig. 6 and Prachar 2012). (The cytoplasmic and nuclear rings are not shown). 
blocks are inbuilt into cytoplasmic and nuclear rings, are consistent with the data published recently (Bui et al. 2013; Szymborska et al. 2013). Moreover, the different quantities of several dynamic nucleoporins in NPC of different cell types even more complicate our present conception about precise NPC architecture (Ori et al. 2013).

Our finding of protein chains copying the curvature of the pore membrane might imply that membrane bending is caused by presumed eight bent pillars. One of the proteins oriented in direction parallel to the transport way (Nup188) was discussed in the recent work of Bui et al. (2013) and probably this one and probably not alone might be engaged in bending the pore membrane as a mechanical support. Moreover, the three NPC rings, spoke, cytoplasmic and nuclear one tied together, can be very strong physical props responsible for membrane bending. Our idea concerning the arrangement of NPC components in the region of the equatorial plane is summarized in the model shown in Fig. 7.

\section{Anchorage of NPC as possible help for bending the pore membrane}

A prerequisite for the insertion of the protein chain into the biological membrane is a proper hydrophobic stretch of amino acids in the given protein sequence. However, such hydrophobic sequences can bind not only lipid chains but they can also bind mutually. The result of these bonds is replacement of a certain area of the membrane with protein chains. Because the binding of proteins and lipids based on hydrophobic properties exhibits certain strength, it could compensate the tension caused by membrane bending. An example of this situation could be the anchorage shown in Fig. $3 b$ and $2 b$. Thus, anchorage itself could also help to keep the proper shape of the pore membrane.

\section{Cytoplasmic and nuclear ring}

In Fig. 1 and Fig. 3, the regions where cytoplasmic and nuclear rings would be localized show too low electron-density and other cellular components and/or transported material is present close to these sites, therefore we did not mark these rings. Generally, many components of the NPC are hydrophobic and consequently they are stained faintly with heavy metal salts. We marked these rings as presumed structures in Fig. 2 on the basis of comparison with three-dimensional images published recently (Maimon et al. 2012). SR is unambiguously determined as a component connected to the NPC barrier.

\section{Lumenal connections}

Small objects called lumenal connections were described recently using the cryo-electron tomography method (Mai- mon et al. 2012). These objects are localized in perinuclear space in the equatorial plane of the NPC. There is but a very low probability to hit into the volume of the small object in the section, whose depth of field is several nanometers only. Analysis of many NPC sections would be required in order to find these objects.

\section{Usage of the terms "protein" and "protein chain" in this article}

There is no proof that the chains observed in high enlargements of the micrographs are really proteins. The only knowledge is that proteins must be present in the given regions. Moreover, chains of polysaccharides could also be present in some regions but we are not able to distinguish them from protein chains or for example from RNA strands. Thus, we simplify the situation and use the name of ubiquitous component regardless of the presence of other biological compounds. We cannot rely on any firm point because such high resolution has not been published to our knowledge so far.

\section{Discontinuity of biological membranes}

The membranes in our micrographs seem to have a "classical" appearance when they are not enlarged (Fig. 3a); this is the light line between two dark lines. The dark lines represent heavy metals bound to phosphate groups; the light line represents lipid chains which are not distinguishable as individual molecules because they are too thin and hydrophobic. As soon as the micrograph is enlarged, many protein chains intersecting the membranes can be discerned. Since we have not seen such a view before, we potentially considered it as a methodological artifact. On the other hand, it is possible to find the regions where the membranes are intact without any insertion (e.g. Fig. 3d). Therefore we concluded this finding to be the property of high resolution and especially of low depth of field. Generally, this finding mirrors the real situation in biological membranes interwoven by integral membrane proteins (see e.g. Schooley et al. 2012). Similarly, we found not only NPC anchorage as a protein complex inserted into the pore membrane but we noted also frequent insertions of large complexes into the inner nuclear membrane from the nuclear side (e.g. Fig. 3e). This is undoubtedly associated with various cellular functions (Blobel 2010). Large complexes inserted into mitochondrial membranes can also be frequently found (Fig. 3f).

\section{Concluding remarks}

In this study, we tried to describe the morphology of the NPC edge in the equatorial plane. This site comprises the SR, NPC anchorage, and we also showed presumed coating module serving for pore membrane bending. High resolution transmission electron microscopy was used for these purposes. We obtained micrographs with a resolution reach- 
ing less than $1 \mathrm{~nm}$, showing individual molecular chains. To our best knowledge, the resolution presented in this work is at least twice higher than ever published in cell section. We are continuing in our efforts to further improve TEM resolution for biological samples, which are sensitive to radiation damage.

Acknowledgements. We are grateful to Mrs. Margita Šulíková for providing cell cultures. This investigation was supported by the Slovak Cancer Research Foundation.

\section{References}

Aitchison J. D., Rout M. P. (2012): The yeast nuclear pore complex and transport through it. Genetics 190, 855-883 http://dx.doi.org/10.1534/genetics.111.127803

Akey C. W., Radermacher M. (1993): Architecture of the Xenopus nuclear pore complex revealed by three-dimensional cryoelectron microscopy. J. Cell Biol. 122, 1-19 http://dx.doi.org/10.1083/jcb.122.1.1

Alber F., Dokudovskaya S., Veenhoff L. M., Zhang W., Kipper J., Devos D., Suprapto A., Karni-Schmidt O., Williams R., Chait B. T., et al. (2007): The molecular architecture of the nuclear pore complex. Nature 450, 695-701 http://dx.doi.org/10.1038/nature06405

Alber F., Dokudovskaya S., Veenhoff L. M., Zhang W., Kipper J., Devos D., Suprapto A., Karni-Schmidt O., Williams R., Chait B. T., et al. (2007): Determining the architectures of macromolecular assemblies. Nature 450, 683-694 http://dx.doi.org/10.1038/nature06404

Amlacher S., Sarges P., Flemming D., van Noort V., Kunze R., Devos D. P., Arumugam M., Bork P., Hurt E. (2011): Insight into structure and assembly of the nuclear pore complex by utilizing the genome of a eukaryotic thermophile. Cell 146, 277-289 http://dx.doi.org/10.1016/j.cell.2011.06.039

Bilokapic S., Schwartz T. U. (2012): 3D ultrastructure of the nuclear pore complex. Curr. Opin. Cell Biol. 24, 86-91 http://dx.doi.org/10.1016/j.ceb.2011.12.011

Blobel G. (2010): Three-dimensional organization of chromatids by nuclear envelope-associated structures. Cold Spring Harb. Symp. Quant. Biol. 75, 545-554 http://dx.doi.org/10.1101/sqb.2010.75.004

Bui K. H., von Appen A., DiGuilio A. L., Ori A., Sparks L., Mackmull M. T., Bock T., Hagen W., Andrés-Pons A., Glavy J. S., Beck M. (2013): Integrated structural analysis of the human nuclear pore complex scaffold. Cell 155, 1233-1243 http://dx.doi.org/10.1016/j.cell.2013.10.055

Debler E. W., Ma Y., Seo H. S., Hsia K. C., Noriega T. R., Blobel G., Hoelz A. (2008): A fence-like coat for the nuclear pore membrane. Mol. Cell 32, 815-826 http://dx.doi.org/10.1016/j.molcel.2008.12.001

DeGrasse J. A., DuBois K. N., Devos D., Siegel T. N., Sali A., Field M. C., Rout M. P., Chait B. T. (2009): Evidence for a shared nuclear pore complex architecture that is conserved from the last common eukaryotic ancestor. Mol. Cell Proteomics 8, 2119-2130
http://dx.doi.org/10.1074/mcp.M900038-MCP200

Devos D., Dokudovskay S., Alber F., Williams R., Chait B. T., Sali A., Rout M. P. (2004): Components of coated vesicles and nuclear pore complexes share a common molecular architecture. PLoS Biol. 2, e380 http://dx.doi.org/10.1371/journal.pbio.0020380

Fernandez-Martinez J., Rout M. P. (2012): A jumbo problem: mapping the structure and functions of the nuclear pore complex. Curr. Opin. Cell Biol. 24, 92-99 http://dx.doi.org/10.1016/j.ceb.2011.12.013

Goldberg M. W., Allen T. D. (1996): The nuclear pore complex and lamina: Three-dimensional structures and interactions determined by field emission in-lens scanning electron microscopy. J. Mol. Biol. 257, 848-865 http://dx.doi.org/10.1006/jmbi.1996.0206

Kirchhausen T. (2012): Bending membranes. Nat. Cell Biol. 14, 906-908 http://dx.doi.org/10.1038/ncb2570

Liu X., Mitchell J. M., Wozniak R. W., Blobel G., Fan J. (2012): Structural evolution of the membrane-coating module of the nuclear pore complex. Proc. Natl. Acad. Sci. U.S.A. 109, 16498-16503 http://dx.doi.org/10.1073/pnas.1214557109

Löschberger A., van de Linde S., Dabauvalle M. C., Rieger B., Heilemann M., Krohne G., Sauer M. (2012): Super-resolution imaging visualizes the eightfold symmetry of gp 210 proteins around the nuclear pore complex and resolves the central channel with nanometer resolution. J. Cell Sci. 125, $570-575$ http://dx.doi.org/10.1242/jcs.098822

Maimon T., Elad N., Dahan I., Medalia O. (2012): The human nuclear pore complex as revealed by cryo-electron tomography. Structure 20, 998-1006 http://dx.doi.org/10.1016/j.str.2012.03.025

Ori A., Banterle N., Iskar M., Andrés-Pons A., Escher C., Bui H. K., Sparks L., Solis-Mezarino V., Rinner O., Bork P., Lemke E. A. Beck M. (2013): Cell type-specific nuclear pores: a case in point for context-dependent stoichiometry of molecular machines. Mol. Syst. Biol. 9, 648 http://dx.doi.org/10.1038/msb.2013.4

Prachar J. (2010): Mouse and human mitochondrial nucleoid--detailed structure in relation to function. Gen. Physiol. Biophys. 29, 160-174 http://dx.doi.org/10.4149/gpb_2010_02_160

Prachař J. (2012): Structural features of transversal barrier in central channel of nuclear pore complex. J. Exp. Cell. Res. $318,614-622$ http://dx.doi.org/10.1016/j.yexcr.2012.01.015

Schooley A., Vollmer B. Antonin W. (2012): Building a nuclear envelope at the end of mitosis: coordinating membrane reorganization, nuclear pore complex assembly, and chromatin de-condensation. Chromosoma 121, 539-554 http://dx.doi.org/10.1007/s00412-012-0388-3

Speese S. D., Ashley J., Jokhi V., Nunnari J., Barria R., Li Y., Ataman B., Koon A., Chang Y. T., Li. Q., et al. (2012): Nuclear envelope budding enables large ribonucleoprotein particle export during synaptic Wnt signaling. Cell 149, 832-846 http://dx.doi.org/10.1016/j.cell.2012.03.032 
Szymborska A., de Marco A., Daigle N., Cordes V. C., Briggs J. A. G., Ellenberg J. (2013): Nuclear pore scaffold structure analyzed by super-resolution microscopy and particle averaging. Science 341, 655-658

http://dx.doi.org/10.1126/science.1240672

Unwin P. N., Milligan R. A. (1982): A large particle associated with the perimeter of the nuclear pore complex. Cell Biol. 93, 63-75 http://dx.doi.org/10.1083/jcb.93.1.63

Weis K. (2002): Nucleocytoplasmic transport: cargo trafficking across the border. Curr. Opin. Cell. Biol. 14, 328-335 http://dx.doi.org/10.1016/S0955-0674(02)00337-X
Wente S. R. (2000): Gatekeepers of the nucleus. Science 288, 1374-1377 http://dx.doi.org/10.1126/science.288.5470.1374

Yang Q., Rout M. P. Akey C. W. (1998): Three-dimensional architecture of the isolated yeast nuclear pore complex: functional and evolutionary implications, Mol. Cell 1, 223-234 http://dx.doi.org/10.1016/S1097-2765(00)80023-4

Received: January 9, 2014

Final version accepted: March 25, 2014

First published online: August 22, 2014 